\title{
Densidad electrónica del gas en la galaxia NGC 3310 con PYNEB
}

\author{
Adan Artola ${ }^{1, *}$ \\ ${ }^{1}$ Máster, Departamento de Astronomía y Astrofísica de la UNAH \\ DOI: https://doi.org/10.5377/ce.v11i2.8677
}

\begin{abstract}
RESUMEN
Presentamos la reducción y análisis del espectro óptico $(\lambda 3600-\lambda 7000 \AA$ A $)$ de la galaxia en espiral con brote estelar NGC 3310. El análisis de densidad electrónica realizado muestra un valor de $n_{e}=$ $167.85 e^{-} / \mathrm{cm}^{3}$ para la zona estudiada. Calculamos el flujo y el ancho equivalente de las líneas de emisión y absorción más intensas de la galaxia. Discutimos el origen de dichas líneas y el proceso de reducción usado para obtenerlas.
\end{abstract}

Palabras clave: NGC 3310, espectroscopia, análisis de líneas, ancho equivalente, flujo.

\begin{abstract}
In this document we present results of the analysis and reduction of the optical spectrum $(\lambda 3600-$ $\lambda 7000 \AA$ ) of the NGC 3310 starburst galaxy. The electronic density analisys shows a value of $n_{e}=$ $167.85 e^{-} / \mathrm{cm}^{3}$ for the studied region. We compute flux and equivalent width of the strongest emission and absortion lines of galaxy. We discuss the origin of those lines and the reduction process used to obtain them.
\end{abstract}

Keywords: NGC 3310, spectroscopy, line analysis, equivalent width, flux.

\section{Introducción}

NGC 3310 es una galaxia con brote estelar clasificada como SAB(r)bc peculiar de luminosidad clase II-III, con dos brazos en espiral. Sus coordenadas son $\alpha_{2000}=10^{\mathrm{h}} 38^{\mathrm{m}} 45.9^{\mathrm{s}}, \delta_{2000}=+53^{\circ} 30^{\prime} 12^{\prime \prime}$ (de Vaucouleurs et al., 1991). Esta galaxia es un buen ejemplo de baja metalicidad en general (Pastoriza et al., 1993), con una alta tasa de formación estelar (> $12 \mathrm{M}_{\odot} \mathrm{yr}^{-1}$ ) y colores muy azules (Telesco y Gatley, 1984). La parte central de la galaxia muestra un anillo de formación estelar de $8-12$ seg de arco de diámetro que contiene más de 100 pequeñas regiones de formación estelar con edades $<10$ Myr para las agrupaciones más grandes (Elmegreen et al., 2002).

Un análisis espectroscópico realizado en (Andrillat y Collin-Souffrin, 1976) muestra una razón de abundancia $\mathrm{A}(\mathrm{O}) / \mathrm{A}(\mathrm{N})$ igual a 3 veces el valor normal, sobreabundancia de oxígeno mas alta que lo normal, y una temperatura media baja en la región de emisión de [OII], siendo esta del rango de $5000<T<7000 \mathrm{~K}$. Observaciones espectro-fotométricas en el óptico y el infrarrojo cercano $(\lambda 3600-\lambda 9600 \AA)$ de seis regiones HII, análisis de abundancias y modelos de foto-ionización, indican que mientras el disco circumnuclear y

*adan.artola@unah.edu.hn 
las regiones HII tienen baja metalicidad $\left(0.2-0.4 Z_{\odot}\right)$, el núcleo tiene abundancias solares (Pastoriza et al., 1993).

Se ha realizado la reducción del espectro de alta calidad de NGC 3310, sobre un amplio rango espectral ( $\lambda 3600-\lambda 7000 \AA$ \&), lo que nos ha permitido obtener el ancho equivalente y flujo de las líneas de emisión y absorción en el mismo y con estos valores calcular las razones entre $\mathrm{H}_{\beta}$ para cada línea.

El espectro de NGC 3310 (ver figura 1), muestra líneas intensas de emisión, similares a las de una región HII. las líneas del [OII] ( $\lambda 3726$ y $\lambda 3729 \AA$ Å) se encuentran mezcladas, lo que dificulta el cálculo de abundancias y temperaturas. La parte azul del espectro en la zona del salto de Balmer presenta líneas de absorción intensas provocadas por términos de alto orden en la serie de Balmer.

En la sección 2 se describe la instrumentación para la observación. En la sección 3 se muestran , los resultados, el análisis y la discusión sobre el origen posible de las líneas de emisión y absorción, así como los valores de flujo encontrados para cada uno de los mismos. En la sección 4 se muestra la discusión de resultados y conclusiones principales.

\section{Telescopio e instrumentación}

Telescopio Asiago. El telescopio reflector de $1.22 \mathrm{~m}$ es el instrumento histórico del Observatorio Astrofísico Asiago. Propiedad de la Universidad de Padua y es operado por el Departamento de Física y Astronomía (UNIPD-DFA). Ha sido utilizado principalmente para la espectroscopia estelar (S. Ciroi). Los telescopios Asiago (telescopio de $1.22 \mathrm{~m}+1.82 \mathrm{~m}$ Copérnico telescopio Schmidt +67/92 telescopio) ofrecen una gran variedad de instalaciones para la observación, desde la fotometría y astrometría multibanda hasta espectroscopia de alta, media y de baja resolución. El espejo primario tiene una forma parabólica con un diámetro efectivo de $120 \mathrm{~cm}$ y un orificio central de $19.5 \mathrm{~cm}$; posee espejo secundario plano elíptico; puede llegar a $45^{\circ}$ cuando se requiere la configuración Newtoniana.

El telescopio está equipado con un espectrógrafo Boller \& Chivens y una cámara CCD Idus Andor de $512 \times 2048$ pixeles. La escala espacial del instrumento sobre la cámara CCD es de 1.25”/pixel.

\section{Análisis de datos y resultados}

\subsection{Corrección al corrimiento al rojo}

El corrimiento al rojo de la galaxia NGC 3310, se calculó en base al corrimiento promedio de las líneas de $\mathrm{H}_{\alpha} \mathrm{y} \mathrm{H}_{\beta}$ con un valor de $z=0.003213$, la corrección se llevo a cabo utilizando la tarea dopcor de IRAF.

\subsection{Corrección de intensidades por extinción debido a polvo interestelar (reddening)}

En esta corrección se utilizó la curva de Whitford (Whitford, 1958) parametrizada por (Miller y Mathews, 1972), teniendo en cuenta que NGC 3310 es una galaxia con un brote estelar significativo principalmente observado en las región HII en las cercanías de su núcleo. Para calcular la constante de reddening se utilizó una razón de flujos de $\mathrm{H}_{\alpha} / \mathrm{H}_{\beta}=2.86$, como se define en (Osterbrock y Ferland, 1989) para galaxias con índices de ionización significativa, después se utilizó la ley de (Cardelli et al., 1989) asumiendo un valor para $R_{V}=3.1$.

Donde $F\left(\mathrm{H}_{\alpha}\right) / F\left(\mathrm{H}_{\beta}\right)$ son los flujos de $\mathrm{H}_{\alpha}$ y $\mathrm{H}_{\beta}$ medidos en el espectro de NGC 3310. Para realizar la corrección se utilizó el comando deredden de IRAF. Las razones entre las intensidades de las líneas del hidrógeno y los datos en (Pastoriza et al., 1993) indican que los datos se redujeron correctamente. 


\subsection{Flujo y ancho equivalente de las líneas}

Hemos utilizado dos maneras diferentes de integrar las intensidades de las líneas:

1. Si las líneas tenían un perfil simple de solo emisión o absorción (ver figura 2.a). se midieron con el comando doble e de splot en IRAF y el FWHM tomado con el comando doble $\mathrm{k}$.

2. Cuando dos o más líneas de emisión se encontraron mezcladas (ver figura 2.b) se utilizó el comando doble d el cual permitió realizar un ajuste gausiano a cada línea mezclada, para la toma de datos no se tomo en cuenta el ajuste de ruido de fondo.

4. Para las líneas en emisión y absorción (ver figura 3) mezcladas se utilizó el método de (1).

Para la medición del error en la integración del flujo, se utilizó la ecuación 3.1:

$$
\sigma_{f}=\frac{F W H M * \sigma_{b}}{F(\lambda)}
$$

Donde $\sigma_{f}$ es el error en el flujo medido, $\sigma_{b}$ es el $\mathrm{rms}$ del ruido de fondo cerca de la línea medida (este dato fue tomado con el comando doble m de splot), $F W H M$ es el Ancho Total a Mitad de Máximo del ajuste gausiano a la línea y $F(\lambda)$ es el flujo medido para esa línea.

\subsection{Líneas encontradas}

NGC 3310 es una galaxia con brote estelar, con múltiples regiones de formación estelar incluso en su núcleo, esto hace que su espectro contenga líneas de emisión similares a las obtenidas en grandes regiones HII. Dentro de las líneas que podemos encontrar con $T_{e}=10^{4} \mathrm{~K}$, se encuentran las líneas de recombinación del Hidrógeno $\left(\mathrm{H}_{\alpha}, \mathrm{H}_{\beta}, \mathrm{H}_{\gamma}, \mathrm{H}_{\delta}, \mathrm{H}_{\epsilon} \mathrm{y} \mathrm{H}_{\zeta}\right)$ y Helio, las cuales son debidas a la recombinación electrón-ion, con el electrón capturado en un alto nivel de Rydberg y una transición subsecuente en cascada a otros niveles energéticos o al estado base, ejemplo de esto es la línea $\mathrm{H}_{\alpha}$ y la línea $\lambda 5876$ del HeI (Pradhan y Nahar, 2011).

Las líneas de absorción provocadas por términos de alto orden en la serie de Balmer se deben al espectro de estrellas de tipo temprano O, B, A, las cuales pueden dominar el espectro de las galaxias con brote estelar (Gonzalez Delgado et al., 1999).

Dos de las líneas más útiles que podemos encontrar en regiones HII para derivar las propiedades físicas del gas son las líneas prohibidas del [OII] ( $\lambda 3726$ y $\lambda 3729 \AA$ A y el [SII] $(\lambda 6717$ y $\lambda 6731 \AA$ ^). Estas transiciones prohibidas se dan debido a que en estas regiones la densidad es extremadamente baja por lo que la emisión depende principalmente del decaimiento radiativo, estas dos líneas se forman en pares de estructura fina "dobletes", y son los mejores diagnósticos de densidad debido a que ocurren a los extremos azul y rojo del espectro (Pradhan y Nahar, 2011). Es de recalcar que en nuestro espectro las líneas [OII] ( $\lambda 3726$ y $\lambda 3729 \AA$ ) se encuentran mezcladas en una sola línea, por lo que no se ha podido obtener su intensidad por separado. En un proceso similar al [OII] y [NII], se obtienen las líneas prohibidas ( $\lambda 4959$ y $\lambda 5007 \AA$ A del [OIII] y las ( $\lambda 6548$ y $\lambda 6584 \AA$ A $)$ del [NII], pero sus transiciones de estructura fina se encuentran separadas por espacios mayores. Las líneas quedan separadas por rangos más grandes de longitudes de onda, y por lo que se dice que son sensibles a la temperatura (Dalgarno y Layzer, 1987).

En la tabla 1 se muestran el flujo en [ergs $\mathrm{cm}^{-2} \mathrm{~s}^{-1}$ ], ancho equivalente en $[\AA ̊$ ] y el error de las líneas medidas se encuentra. Hay señalar la falta de continuo en la medición de las líneas en absorción, por lo que su $\sigma_{f}$ no pudo ser medido. En la tabla 2 se muestran las transiciones atómicas que dan lugar a las líneas encontradas. 


\subsection{Densidad Electrónica}

La densidad electrónica se obtuvo de las líneas de [SII] ( $\lambda 6716$ y $\lambda 6731 \AA$ A ), para calcular este valor se utilizó la función getTemDen de Pyneb, utilizando una temperatura de $10^{4} \mathrm{~K}$, que es tipica para nebulosas con abundancias normales (Osterbrock y Ferland, 1989). Esta función nos permitió obtener un valor de densidad electrónica de $n_{e}=167.85 e^{-} / \mathrm{cm}^{3}$ este valor es parecido al obtenido para regiones HII en (Pastoriza et al., 1993).

\section{Discusión y conclusiones}

El espectro estudiado presenta una densidad electrónica aproximada de $n_{e}=167.85 e^{-} / \mathrm{cm}^{3}$. Este valor se calcula para una temperatura típica $T=10^{4} \mathrm{~K}$ de regiones HII.

La zona estudiada tiene una muy alta proporción de estrellas $\mathrm{O}, \mathrm{B}, \mathrm{A}$, debido a que fuertes componentes de hidrógeno en absorción fueron observadas en la región azul del espectro.

\section{Agradecimientos}

Agradezco con mi más sincero respeto al Dr. Jacopo Fritz, quien facilito los datos e hizo las revisiones necesarias, lo que me permitió lograr los objetivos propuestos.

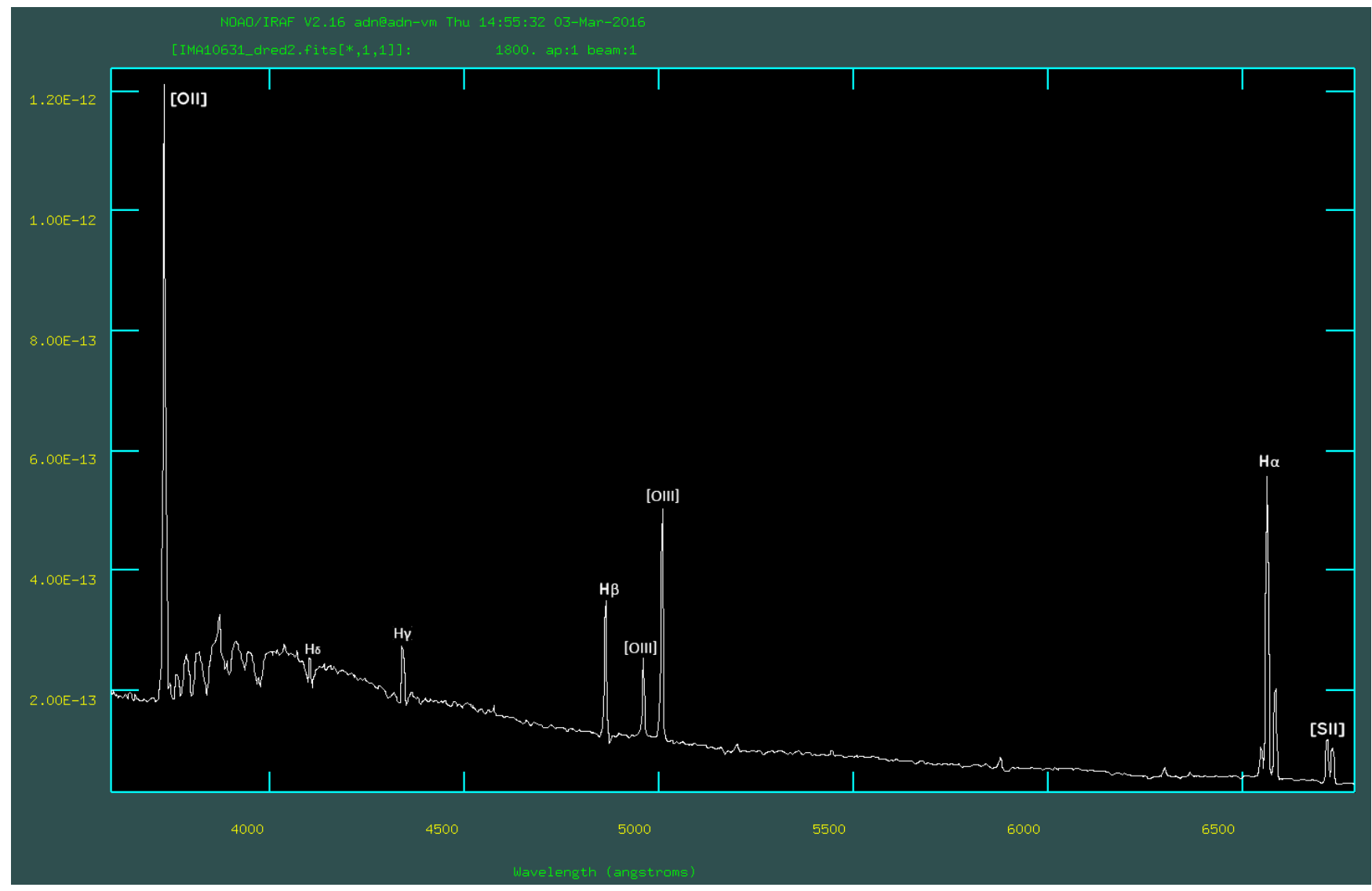

Figura 1: Espectro estudiado de la Galaxia NGC 3310. Fuente: Imagen propia. 


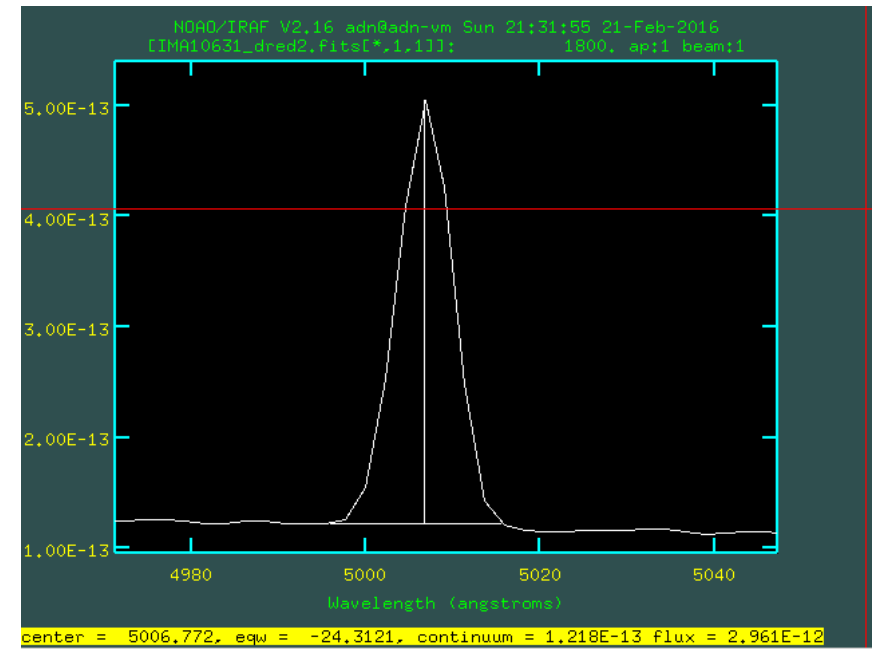

(a)

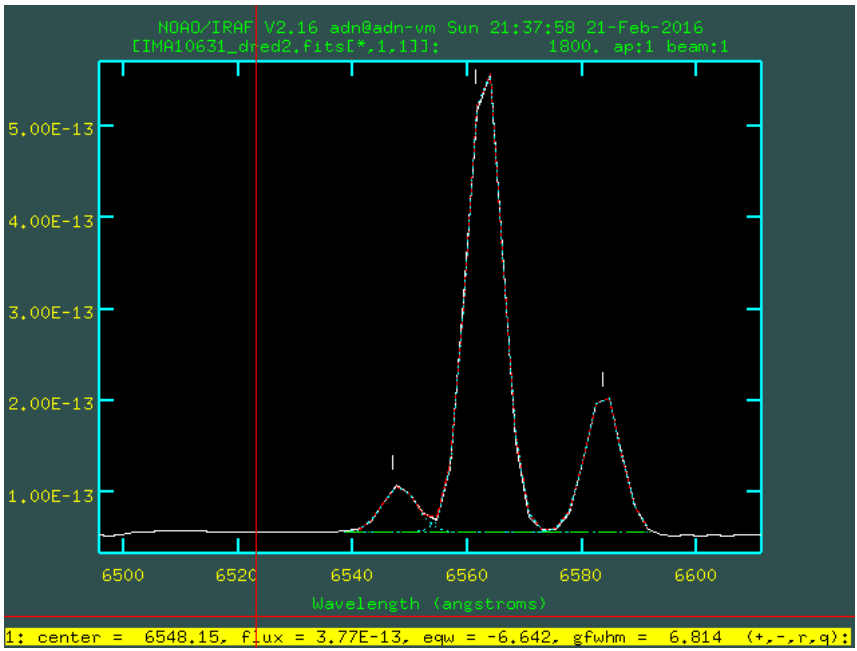

(b)

Figura 2: (a) Línea simple de emisión medida con el comando doble e de IRAF. (b), Línea mezclada de emisión medida con el comando doble d de SPLOT. Fuente: Imagen propia.

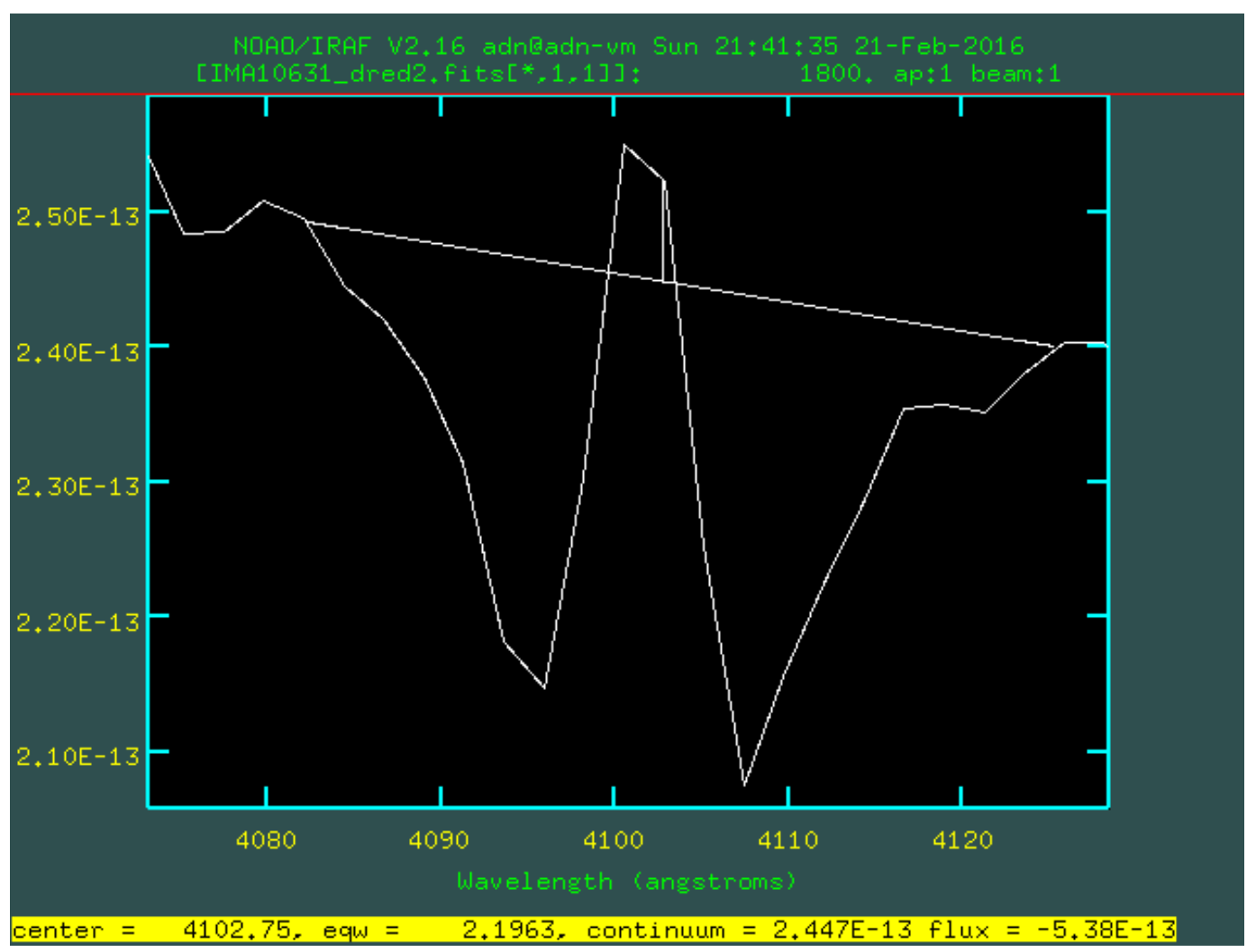

Figura 3: Línea de absorción y emisión medida con el método 1. Fuente: imagen propia . 
Tabla 1: Intensidades de las líneas encontradas en el espectro de NGC 3310

\begin{tabular}{lccccc}
\hline Línea & $\lambda(\AA)$ & Flujo $\left(\times 10^{-14}\right)$ & Ancho Equivalente & $\left(\lambda / \mathrm{H}_{\beta}\right) \times 100$ & $\sigma_{F}$ \\
\hline$[\mathrm{OII}]$ & 3739.74 & 105.00 & -47.51 & 418.33 & \pm 1.2 \\
$\mathrm{H} 10$ & 3762.22 & - & 1.31 & - & - \\
$\mathrm{H} 9$ & 3783.44 & - & 2.21 & - & - \\
$\mathrm{H}_{\eta}$ & 3847.34 & - & 4.47 & - & - \\
$\mathrm{H}_{\zeta}$ & 3902.15 & - & 3.87 & - & - \\
$\mathrm{CaII}(\mathrm{K})$ & 3945.81 & - & 0.97 & - & - \\
$\mathrm{H}_{\epsilon}$ & 3982.96 & - & 4.29 & - & - \\
$\mathrm{H}_{\delta}$ & 4114.37 & - & 1.63 & - & - \\
$\mathrm{H}_{\gamma}$ & 4354.72 & 4.48 & -2.80 & 17.83 & \pm 6.97 \\
$\mathrm{H}_{\beta}$ & 4887.17 & 25.10 & -9.71 & 100.00 & \pm 1.25 \\
{$[\mathrm{OIII}]$} & 4974.76 & 23.00 & -8.94 & 91.63 & \pm 0.88 \\
{$[\mathrm{OIII}]$} & 5022.89 & 64.40 & -25.64 & 256.57 & \pm 0.36 \\
{$[\mathrm{OI}]$} & 6321.49 & 5.34 & -3.14 & 21.27 & \pm 3.74 \\
{$[\mathrm{NII}]$} & 6569.21 & 12.10 & -6.62 & 48.20 & \pm 2.65 \\
$\mathrm{H}_{\alpha}$ & 6584.14 & 121.00 & -71.55 & 482.072 & \pm 0.28 \\
{$[\mathrm{NII}]$} & 6604.83 & 39.10 & -21.98 & 155.78 & \pm 0.85 \\
{$[\mathrm{SII}]$} & 6738.59 & 18.90 & -11.95 & 75.29 & \pm 1.04 \\
{$[\mathrm{SII}]$} & 6752.83 & 15.30 & -9.74 & 60.96 & \pm 1.31 \\
\hline
\end{tabular}

Tabla 2: Transiciones causantes de las líneas de emisión en el espectro de NGC 3310

\begin{tabular}{lcc}
\hline Línea & $\lambda(\AA)$ & Transición \\
\hline$[\mathrm{OII}]$ & $3726.03,3728.8$ & $2 s^{2} 2 p^{3}\left({ }^{4} S_{3 / 2}^{0}-{ }^{2} D_{3 / 2,5 / 2}^{0}\right)$ \\
$\mathrm{H} 10$ & 3750.15 & $12-2$ \\
$\mathrm{H} 9$ & 3770.63 & $11-2$ \\
$\mathrm{H}_{\eta}$ & 3835.38 & $9-2$ \\
$\mathrm{H}_{\zeta}$ & 3889.05 & $2-8$ \\
$\mathrm{CaII}(\mathrm{K})$ & 3790.9 & $3 p^{6} 4 s^{2} S_{\frac{1}{2}}-3 p^{6} 4 p^{o} S_{\frac{3}{2}, \frac{1}{2}}$ \\
$\mathrm{H}_{\epsilon}$ & 3970.07 & $2-6$ \\
$\mathrm{H}_{\delta}$ & 4101.73 & $2-5$ \\
$\mathrm{H}_{\gamma}$ & 4340.46 & $2-4(3 s, 3 p s 3 d-4 s, 4 p, 4 d, 4 f)$ \\
$\mathrm{H}_{\beta}$ & 4861.32 & $2 s^{2} 2 p^{2}\left({ }^{3} P_{1,2}-{ }^{1} D_{2}\right)$ \\
{$[\mathrm{OIII}]$} & $4958.91,5006.84$ & $2 s^{2} 2 p^{4}\left({ }^{3} P_{2,1}-{ }^{1} D_{2}\right)$ \\
{$[\mathrm{OI}]$} & 6302.05 & $2 s^{2} 2 p^{2}\left({ }^{3} P_{1,2}-{ }^{1} D_{2}\right)$ \\
{$[\mathrm{NII}]$} & $6548.04,6583.46$ & $2-3(2 s, 2 p-3 s, 3 p, 3 d)$ \\
$\mathrm{H}_{\alpha}$ & 6562.8 & $3 s^{2} 3 p^{3}\left({ }^{4} S_{3 / 2}^{0}-{ }^{2} D_{5 / 2,3 / 2}^{0}\right)$ \\
{$[\mathrm{SII}]$} & $6716.44,6730.81$ &
\end{tabular}




\section{Referencias}

Andrillat, Y. y Collin-Souffrin, S. (1976). Abundances and physical conditions in the nucleus of the peculiar galaxy NGC 3310. Astronomy and Astrophysics, 49:251-257.

Cardelli, J., Clayton, G., y Mathis, J. (1989). The relationship between infrared, optical, and ultraviolet extinction. Astrophysical Journal, 345:245-256.

Dalgarno, A. y Layzer, D., editores (1987). Spectroscopy of astrophysical plasmas. Cambridge University Press.

de Vaucouleurs, G., de Vaucouleurs, A., Corwin, Jr., H., Buta, R., Paturel, G., y Fouqué, P. (1991). Third Reference Catalogue of Bright Galaxies. Volume I: Explanations and references. Volume II: Data for galaxies between $0^{h}$ and $12^{h}$. Volume III: Data for galaxies between $12^{h}$ and $24^{h}$. P.Springer, New York, NY (USA).

Elmegreen, D. M., Chromey, F. R., McGrath, E. J., y Ostenson, J. M. (2002). Circumnuclear star formation in the spiral galaxy NGC 3310. The Astronomical Journal, 123(3):1381-1388.

Gonzalez Delgado, R. M., Leitherer, C., y Heckman, T. M. (1999). Synthetic spectra of h balmer and heiabsorption lines. ii. evolutionary synthesis models for starburst and poststarburst galaxies. The Astrophysical Journal Supplement Series, 125(2):489-509.

Miller, J. y Mathews, W. (1972). The Recombination Spectrum of the Planetary Nebula NGC 7027. Astrophysical Journal, 172:593.

Osterbrock, D. y Ferland, G. (1989). Astrophysics of gaseous nebulae and active galactic nuclei. Sausalito, California:University Science Books.

Pastoriza, M. G., Dottori, H. A., Terlevich, E., Terlevich, R., y Díaz, A. I. (1993). Optical and near-IR spectrophotometry of the galaxy NGC 3310. Monthly Notices of the Royal Astronomical Society, 260(1):177190.

Pradhan, A. y Nahar, S. (2011). Atomic Astrophysics and Spectroscopy. Cambridge University Press.

Telesco, C. y Gatley, I. (1984). Ongoing star formation in NGC 3310 - an infrared perspective. Astrophysical Journal, 284:557-564.

Whitford, A. (1958). The law of interstellar reddening. Astronomical Journal, 63:201-207. 